\title{
BMJ Global Health Increasing demand for school counselling through a lay counsellor-delivered classroom sensitisation intervention: a stepped-wedge cluster randomised controlled trial in New Delhi, India
}

Rachana Parikh (D) , ,2,3 Adriaan Hoogendoorn, ${ }^{4,5}$ Daniel Michelson, ${ }^{6}$ Jeroen Ruwaard, ${ }^{4,5}$ Rhea Sharma, ${ }^{2}$ Bhargav Bhat, ${ }^{7}$ Kanika Malik, ${ }^{2}$ Rooplata Sahu, ${ }^{2}$ Pim Cuijpers, ${ }^{8}$ Vikram Patel $^{7,9,10}$

To cite: Parikh $\mathrm{R}$, Hoogendoorn A, Michelson D, et al. Increasing demand for school counselling through a lay counsellor-delivered classroom sensitisation intervention: a steppedwedge cluster randomised controlled trial in New Delhi, India. BMJ Global Health 2021;6:e003902. doi:10.1136/ bmjgh-2020-003902

Handling editor Soumitra S Bhuyan

JR deceased

Received 4 September 2020 Accepted 27 May 2021
Check for updates

(c) Author(s) (or their employer(s)) 2021. Re-use permitted under CC BY-NC. No commercial re-use. See rights and permissions. Published by BMJ.

For numbered affiliations see end of article.

Correspondence to Dr Vikram Patel; vikram_patel@hms.harvard.edu

\section{ABSTRACT}

Introduction We evaluated a classroom-based sensitisation intervention that was designed to reduce demand-side barriers affecting referrals to a school counselling programme. The sensitisation intervention was offered in the context of a host trial evaluating a low-intensity problem-solving treatment for common adolescent mental health problems.

Methods We conducted a stepped-wedge, cluster randomised controlled trial with 70 classes in 6 secondary schools serving low-income communities in New Delhi, India.The classes were randomised to receive a classroom sensitisation session involving a brief video presentation and moderated group discussion, delivered by a lay counsellor over one class period (intervention condition, IC), in two steps of 4 weeks each. The control condition (CC) was whole-school sensitisation (teacher-meetings and whole-school activities such as poster displays). The primary outcome was the proportion of students referred into the host trial. Secondary outcomes were the proportion of students who met mental health caseness criteria and the proportion of self-referred adolescents.

Results Between 20 August 2018 and 9 December 2018,835 students $(23.3 \%$ of all students) were referred into the host trial. The referred sample included 591 boys $(70.8 \%)$, and had a mean age of 15.8 years, $S D=0.06 ; 194$ students $(31.8 \%$ of 610 with complete data) met mental health caseness criteria. The proportion of students referred in each trial conditionwas significantly higher in the IC $(\mathrm{IC}=21.7 \%, \mathrm{CC}=1.5 \%, 0 \mathrm{R}=111.36,95 \% \mathrm{Cl} 35.56$ to $348.77, p<0.001)$. The proportion of self-referred participants was also higher in the IC $(\mathrm{IC}=98.1 \%$, $\mathrm{CC}=89.1 \%$, Pearson $\left.\chi^{2}(1)=16.92, \mathrm{p}<0.001\right)$. Although the proportion of referred students meeting caseness criteria was similar in both conditions ( $\mathrm{IC}=32.0 \%$ vs $\mathrm{CC}=28.1 \%$ ), the proportion weighted for the total student population was substantially higher in the IC $(\mathrm{IC}=5.2 \%, \mathrm{CC}=0.3 \%, \mathrm{OR}=52.39,95 \% \mathrm{Cl} 12.49$ to $219.66, p<0.001)$.

\section{Key questions}

What is already known?

- Low mental health literacy and high stigma restrict adolescents' help-seeking for mental health problems.

- Previous studies suggest that demand-side interventions are likely to increase access to menta healthcare when targeted directly at young people rather than focusing primarily on parents and other adult gatekeepers.

- The existing evidence base is derived almost entirely from high-income countries, limiting inferences to other contexts.

What are the new findings?

- We report findings from the first randomised controlled trial of a youth-focused intervention that aims to increase demand for school-based mental healthcare in a low-resource context.

- As compared with a whole-school sensitisation programme (which targeted teachers and school principals, with adolescent engagement limited to poster displays and installation of drop boxes for receiving referrals), a single classroom sensitisation session (involving a video presentation and group discussion with a lay counsellor) led to a large increase in demand for school-based counselling.

- The vast majority of help-seeking adolescents were self-referred.

- A greater proportion of the help-seeking students who met mental health caseness criteria originated from the sensitised classes.

Conclusion A single, lay counsellor-delivered, classroom sensitisation session increased psychological help-seeking for common mental health problems among secondary school pupils from urban, low-income communities in India.

Trial registration number NCT03633916. 


\section{Key questions}

What do the new findings imply?

- Compared with whole-school sensitisation, this study demonstrates that a brief, student-targeted intervention, delivered at the classroom level using contextually sensitive content and facilitated by a lay counsellor, can greatly increase demand for mental healthcare among socioeconomically disadvantaged adolescents.

- In conjunction with evidence-based psychological treatment, this approach has the potential to reduce the mental healthcare gap for young people in low-resourced and potentially also in highresourced settings.

\section{INTRODUCTION}

Help-seeking for adolescent mental health problems is low in all contexts, but particularly so in low-income and middle-income countries (LMICs). ${ }^{1-4} \mathrm{~A}$ range of demand-side barriers have been described in the international literature. These include low mental health literacy (associated with difficulties in self-identification of symptoms and poor knowledge of mental health services); stigma; a preference towards self-reliance and informal sources of help; perceived ineffectiveness of treatments; concerns regarding confidentiality; and negative past experiences with mental health services. ${ }^{1-5}$ Such factors are compounded by systemic barriers such as scarcity of acceptable and effective mental health services and relatively high associated costs. ${ }^{1-5}$ Schools are a recommended platform to improve adolescents' access to effective mental healthcare, with the potential to address a number of these barriers. However, even when schoolbased mental health services are available, adolescents may be reluctant to seek help. ${ }^{6}$

There has been growing interest in developing and testing interventions to increase the demand for mental healthcare by adolescents, especially in the past decade. However, a recent meta-analysis reported no effects of demand-side interventions on formal help seeking for adolescent mental health problems; notably, most of the interventions included were targeted towards caregivers or gatekeepers rather than adolescents directly. ${ }^{7}$ Interventions targeting adolescents themselves, such as universal school-based mental health awareness programmes, have been shown to improve mental health literacy and influence more positive help-seeking attitudes and intentions among adolescents. These variables are generally assumed to promote actual help seeking. ${ }^{8-10}$ We found seven studies (none in LMICs) that assessed the impact of such interventions on actual help-seeking behaviour, four of which were conducted in schools ${ }^{11-14}$ and focused variously on mental health literacy ${ }^{11}$ and suicide literacy. ${ }^{12-14}$ None of these school-based intervention studies showed any positive impact on help-seeking behaviour.

India is home to the largest number of adolescents in any single country and a growing policy attention has been devoted to mental health in this group, with a particular focus on school-based provision of mental healthcare. ${ }^{15}$ Given the very low coverage of school mental health services in India and an estimated treatment gap for common mental disorders in adults of $85 \%,{ }^{16}$ we speculate that the unmet mental health needs for adolescents exceed $90 \%$.

The PRemIum for aDolEscents (PRIDE) programme aims to develop and evaluate a stepped-care intervention model for adolescents with common mental health problems in low-income schools in India. The PRIDE steppedcare architecture involves two sequential psychological interventions of incremental intensity: the first step is a brief low-intensity problem-solving intervention, ${ }^{17}$ while the second step is a higher-intensity psychological treatment that is offered to those students who do not respond to the problem-solving intervention. ${ }^{18}$ An iterative process of intervention development highlighted access barriers such as low mental health literacy and concerns regarding confidentiality in the target population, with consequent needs for clear and non-stigmatising information about the purpose and intended recipients of school counselling services, referral processes and assurances about confidentiality. ${ }^{19}{ }^{20}$ To address these barriers, we designed a universal classroom-level interventionaimed at stimulating help-seeking behaviour among secondary school pupils, ${ }^{9}$ and then evaluated its impacts in an embedded linked trial to a host trial of the first-step problem-solving intervention. ${ }^{21}$ This paper presents the results of this embedded sensitisation trial, and complements the findings of the host trial. ${ }^{22}$

The primary objective of the sensitisation trial was to evaluate the impact of a classroom sensitisation session (intervention condition, IC), over and above wholeschool sensitisation activities (control condition, CC), on the proportion of adolescents referred into the host trial as a function of the total sampling frame in each condition. We hypothesised that the IC would be associated with a higher proportion of students referred from the corresponding school population. The secondary objectives were to assess the effects of the intervention on (1) the proportion of students who met mental health "caseness' criteria (as required to participate in the host trial) and (2) the proportion of students who were self-referred. We also sought to explore whether there would be any differences in the severity of symptoms and symptom subtypes among help-seeking students after exposure to the respective sensitisation conditions.

\section{METHODS \\ Study design}

We conducted a stepped-wedge cluster randomised controlled trial (SW-CRCT) ${ }^{23}$ with two sequences and three time periods in six Government-run secondary schools in New Delhi, India. Three were boys' schools, two were girls' schools and one was co-educational (mixed boys and girls), all catering to low-income communities. The design has been described in detail in the published study protocol. ${ }^{21}$ In brief, we randomly 


\begin{tabular}{|l|l|l|l|l|}
\hline \multicolumn{2}{|l|}{} & \multicolumn{1}{|c|}{ Step 1 } & \multicolumn{1}{|c|}{ Step 2 } & $\begin{array}{l}\text { Sequence of receiving } \\
\text { treatments* }\end{array}$ \\
\hline $\begin{array}{l}\text { Sequence 1 } \\
\text { (1-35 classes) }\end{array}$ & $\begin{array}{l}\text { Control } \\
\text { condition }\end{array}$ & $\begin{array}{l}\text { Intervention } \\
\text { condition }\end{array}$ & $\begin{array}{l}\text { Intervention } \\
\text { condition } \\
\text { (Continued } \\
\text { effect) }\end{array}$ & 011 \\
\hline $\begin{array}{l}\text { Sequence 2 } \\
\text { (36-70 } \\
\text { classes) }\end{array}$ & $\begin{array}{l}\text { Control } \\
\text { condition }\end{array}$ & Control condition & $\begin{array}{l}\text { Intervention } \\
\text { condition }\end{array}$ & 001 \\
\hline $\begin{array}{l}\text { Time periods } \\
1^{\text {st }} \text { time } \\
\text { period } \\
\text { (4 weeks) }\end{array}$ & $\begin{array}{l}2^{\text {nd }} \text { time period } \\
\text { (4 weeks) }\end{array}$ & $\begin{array}{l}3^{\text {rd }} \text { time } \\
\text { period } \\
\text { (4 weeks) }\end{array}$ & \\
\hline
\end{tabular}

Notes: The white boxes indicate the group of classes in the control condition comprising of school-level sensitisation activities and the coloured boxes indicate the group of classes in the intervention condition comprising of a single classroom sensitisation session delivered by the lay counsellors and school based researchers. The arrows indicate the implementation of the classroom sensitisation session for the two sequences.

${ }^{*} \mathrm{O}=$ control condition; $1=$ intervention condition

Figure 1 Illustration showing implementation of the control and intervention conditions.

selected 70 classes (sampled from grades 9-12) covering 3587 students, from a pool of 118 classes that had not received classroom sensitisation previously during pilot work. We restricted the number of classes included in the trail to 70 , based on the number of cases we expected by modelling of pilot data, so that the counsellors in the host problem-solving intervention trial could manage the demand. Classes formed natural clusters.

All classes began in the CC (first time period), and subsequently received the intervention in two steps (figure 1). Classes were randomised to one of the two allocation sequences that determined the step in which they received the intervention. Thus, the trial had three time periods of 4 weeks each, and a total duration of 12 weeks.

\section{Randomisation and masking}

Random numbers were generated using the rand() command in Microsoft Excel and assigned to the 118 available classes arranged in strata by school and grade. The final sample of 70 classes was selected through proportional sampling from each of these strata using the smallest value of the assigned random number as a guide. The randomisation sequence for allocating these 70 classes to one of the two sequences was generated (in Excel) using a block size of two (as the numbers of classes in individual strata was often less than 4). The randomisation was completed before the initiation of the trial. Blinding of the researchers was not possible as they were involved in the implementation of sensitisation activities across both the IC and CC. Teachers were not informed about the trial hypotheses so as not to influence their willingness to refer students into the host trial.

\section{Procedures}

Control condition

This comprised a set of whole-school sensitisation activities intended to generate referrals of students with common mental health problems into a counselling service on school premises. These activities were: meetings with school principals and teachers to provide them with structured information about common mental health problems among students and counselling referral criteria/ procedures; displaying posters with information about the counselling service in highly visible locations throughout the school, such as noticeboards, corridors and outside the counsellor's room; and establishing drop-boxes (one per school) with appropriate signage installed near the counsellor's room, for receiving referrals discreetly. A liaison teacher was identified in each of the schools to coordinate these activities. Meetings with the principal were conducted by master's-level psychologists while lay counsellors (also responsible for providing the problem-solving intervention in the host trial) and researchers (responsible for processing referrals and conducting mental health assessments for the current and the host trial) convened meetings with the teachers. The lay counsellors and researchers were Hindi-speaking college graduates. The counsellors had no prior training or experience of mental healthcare.

\section{Intervention condition}

This comprised a single classroom sensitisation session offered in addition to the whole-school sensitisation activities. A typical session was delivered over $20-25 \mathrm{~min}$ (to fit into a typical class period) by a lay counsellor aided by a researcher. The session began with a short animated video (link to video) providing age-appropriate information on common stressors and ways of coping. This was followed by a moderated group discussion to facilitate engagement and clarify understanding of the material. Additionally, adolescents were handed a self-referral form which included normalising information and questionbased prompts to assist with self-identification of mental health problems. Fidelity of the intervention was assessed for $20 \%$ of sessions by an independent assessor using a checklist of 16 observable procedures (eg, accuracy of explanations given about confidentiality, referral pathways and use of self-referral forms; responsiveness to queries raised by students during the class discussion). Each item was evaluated on a three-point Likert scale. A fidelity score (maximum score $=2$ ) was arrived at after averaging the individual scores across all assessed procedures.

Across both the conditions, adolescents could be referred into the host trial through three routes: selfreferral by meeting a counsellor/researcher personally; depositing a referral slip in a drop-box; or referral by a teacher. Consent was obtained through a two-stage process covering both this and the host trial, approved by the Institutional Review Boards of Sangath and Harvard Medical School. Referred adolescents were provided with structured verbal information and a written information sheet about the use of their referral/screening data, with the opportunity to opt-out. The information sheet was retained by adolescents and shared with parents/guardians. Adolescents meeting eligibility criteria for the host 
trial were provided with additional structured verbal and written information and an opt-in procedure was implemented, as outlined in the published protocol. ${ }^{21}$ It was not viable to obtain informed consent from all the 3587 students in the selected classes prior to randomization as the intervention was delivered at classroom level, and the consent process itself was likely to influence the students' help-seeking directly. Steps were taken to ensure that no personally identifiable data were collected before obtaining informed consent.

\section{Outcomes}

Primary outcome: 'Referral proportion' was the proportion of adolescents in the participating classes that were referred for counselling from a particular sensitisation condition.

Secondary outcomes: (1) 'Case-level proportion' was defined in two ways: firstly, the proportion of students who met the criteria for mental health 'caseness' as a fraction of all students in the participating classes (added to the original protocol, before commencing data analysis), and secondly, the proportion of students who met the criteria for mental health 'caseness' as a fraction of referred students only. The criteria for mental health 'caseness' reflected a symptom severity level that met the eligibility criteria for participation in the host trial.

The counselling intervention in the host trial was designed as an early intervention for adolescents with elevated symptoms and associated distress/impairment. Adolescents were eligible to participate in the host trial if they reported (1) Total Difficulties score on the self-reported Strengths and Difficulties Questionnaire $(\mathrm{SDQ})^{24}$ above the locally validated cut-offs of $>/=19$ and $>/=20$ for borderline difficulties among boys and girls respectively, representing the top $20 \%$ of scores obtained from a normative reference sample in Indian youth ${ }^{25}$; (2) score of 2 or more on the SDQ Impact Supplement, indicating clinically significant distress and functional impairment; (3) duration of mental health problems lasting for more than 1 month, assessed on the chronicity item on the SDQ Impact Supplement. The SDQ has been widely used as a measure of psychopathology among children and adolescents globally, including in India. ${ }^{26}$

(2) 'Self-referral proportion' was defined as the proportion of referred adolescents who were self-referred.

Exploratory outcomes:We also assessed the effect of the IC on the following exploratory outcomes among students referred from the particular sensitisation condition: (1) severity of adolescent-reported mental health symptoms on the SDQ Total Difficulties scale; and (2) severity of adolescent-reported symptoms on the SDQ Internalising and Externalising problems sub-scales. These sub-scale scores (range 0-20) were derived from summing scores for Emotional/Peer relationship problems and Conduct/Hyperactivity-inattention problems, respectively.

\section{Analysis}

A power calculation was based on a within-period comparison for an SW-CRCT, ${ }^{27}$ using Stata package 'clustersampsi' and parameters based on pilot data. Using referral proportions of $5 \%$ for the CG and $15 \%$ for the IC, and an intracluster correlation coefficient (ICC) of 0.124 , a pragmatic sample size of 70 classes (average class size of 50 students) provided $92 \%$ power to detect a difference of 10 percentage points (treating the outcome as a binomial variable), at a significance level of 0.05 .

While data for the primary outcome of referral proportion and the secondary outcome of self-referral proportion were available for all of the 835 referred adolescents, assessments for eligibility to participate in the host trial were completed for 610 participating adolescents. Data for individual items on the SDQ were lost for one of these 610 students due to a technical malfunction. Thus, exploratory outcomes-requiring the scores of individual items on the SDQ-were available for 609 adolescents.

We used multiple imputations with a chained equations framework to impute missing data for the 225 referred adolescents who did not participate in the host trial (updated in the statistical methods outlined in the original protocol before commencing data analysis). We first imputed the missing data for the underlying variables of the Internalising and Externalising SDQ subscale scores, Impact score and chronicity of mental health problems. The SDQ Total Difficulties score and eligibility for the host trial were then computed passively from the imputed data for the underlying variables. We used univariate methods with logit for categorical variables, and predictive mean matching (k-nearest-neighbour option=5) for continuous variables. The imputation model included period, allocation sequence, school and grade, as they were associated with trial participation (Table 2). The number of imputations was set to 50 , to account for the high proportion of missing data. ${ }^{28}$

We used generalised estimating equations (GEE) with robust standard errors (measured using the sandwich estimator method) for analysis of the outcomes, a recommended method for analysis of the SW-CRCTs. ${ }^{29}$ The analysis model for calculating marginal probability of the outcomes included parameters for the effect of the classroom sensitisation session for the time periods when the intervention was introduced and for the subsequent time period. We also included parameters for the second and the third time period (added to the initial analysis plan before commencing data analysis) to account for secular trend. We specified the 'exchangeable' correlation structure, later changed to 'independent' when the model did not converge for the outcomes of referral proportion, and eligible proportion. Sensitivity analysis was conducted using GEE for a "within-period comparison' of data from the second period only. ${ }^{27}$

\section{Patient and Public Involvement}

Formative research activities, including literature reviews and intervention design workshops with local and 
international experts, were triangulated with qualitative data from 280 stakeholders, including adolescents, parents, school staff and mental health practitioners. ${ }^{17}$ Structured school-based sensitisation was endorsed by the various stakeholder groups to overcome demandside barriers to engagement in mental health services. ${ }^{19}$ These activities were subsequently tested and refined in pilot work prior to the current trial. ${ }^{17}$ Key modifications included: (1) redesigned content of classroom sessions with stronger emphasis placed on normalisation of adolescent mental health problems and assurances about confidentiality to address concerns about stigma; (2) use of an animated video and group discussion (rather than didactic verbal instructions) to increase student engagement with the classroom session; (3) increased variety of referral methods to facilitate discreet self-referral processes; and (4) use of structured/scripted briefings to standardise information provided to school staff.
RESULTS

\section{Trial flow indicators}

The trial began on 20 August 2018, and was completed on 9 December 2018 after accommodating two short breaks-one for exams between the first and the second time periods and the second for a major Indian festival (Diwali) between the second and third time periods of the trial (figure 2). The total student population in the 70 randomly selected classes was 3587 with a mean class size of 51.2 (95\% CI 47.3 to 55.2); 2036 (56.8\%) were boys. Across the two sequences of IC delivery, there was a balanced distribution with respect to grades (ie, across years 9-12). However, the number of adolescents included in each of the six schools and the gender distribution differed significantly (table 1 ).

Overall, there were 835 referrals $(23.3 \%$ out of 3587 students) from the 70 classes included in this trial (mean referrals per class $=12, \mathrm{SD}=1.55$ ). From these 835

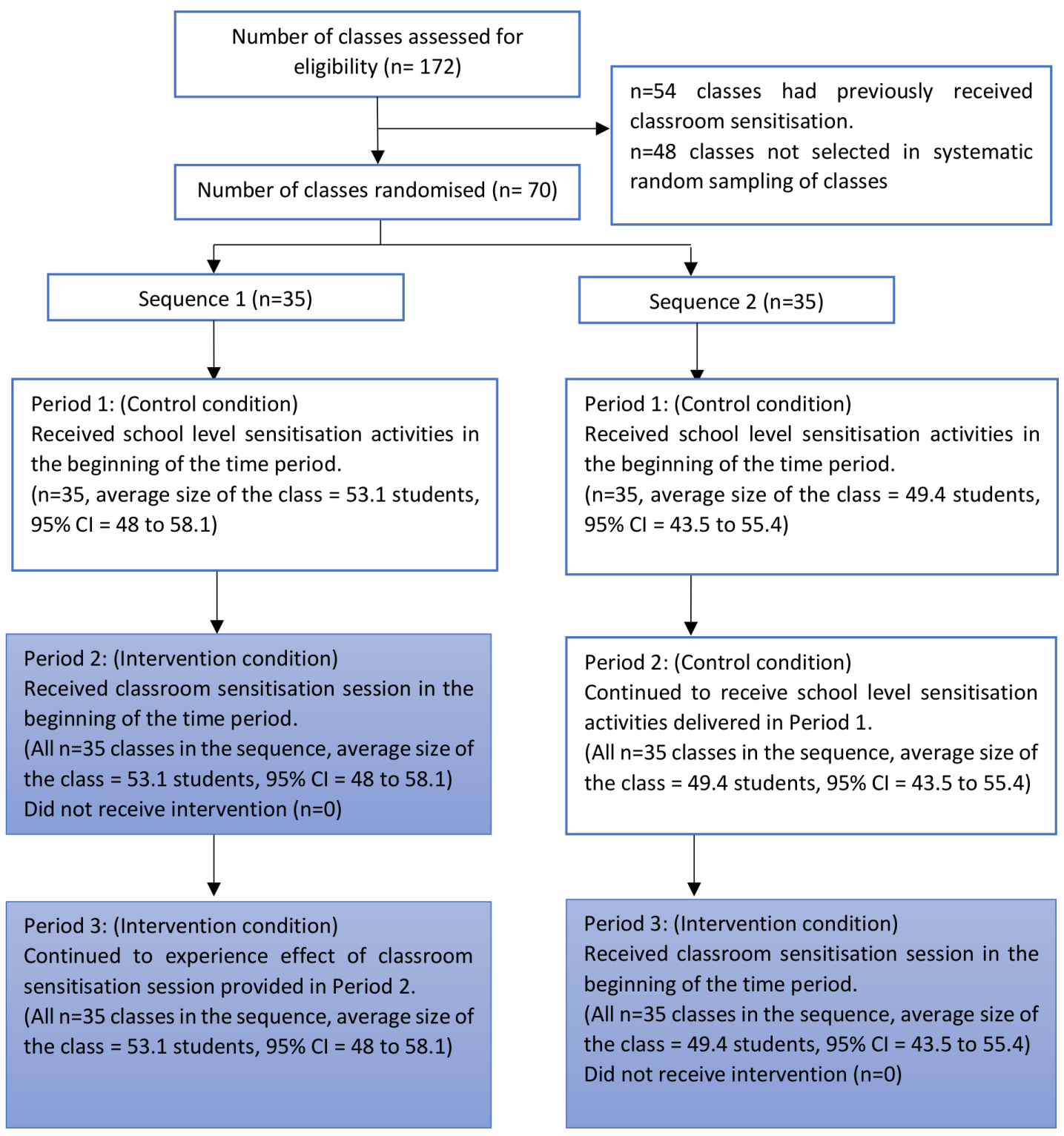

Figure 2 CONSORT flow chart. CONSORT, Consolidated Standards of Reporting Trials . 
Table 1 Characteristics of students in participating classes (clusters) by the allocation sequences

\begin{tabular}{|c|c|c|c|c|c|c|}
\hline & \multirow{2}{*}{$\begin{array}{l}\text { Total } \\
\text { Students } \\
(\mathrm{N}=3587)\end{array}$} & \multicolumn{2}{|c|}{ Sequence $1=35$ classes } & \multicolumn{2}{|c|}{ Sequence $2=35$ classes } & \multirow[b]{2}{*}{$\begin{array}{l}\text { Test statistic, } \\
\text { p value }\end{array}$} \\
\hline & & $\begin{array}{l}\text { Students } \\
(n=1857)\end{array}$ & $\%$ & $\begin{array}{l}\text { Students } \\
(n=1730)\end{array}$ & $\%$ & \\
\hline Gender: & & & & & & Pearson $\chi^{2}$ \\
\hline Male & 2036 & 1106 & 54.3 & 930 & 45.7 & $(1)=12.3, p<0.001$ \\
\hline Female & 1551 & 751 & 48.4 & 800 & 51.6 & \\
\hline Grade: & & & & & & Pearson $\chi^{2}$ \\
\hline 9th & 1614 & 831 & 51.5 & 783 & 48.5 & $(3)=0.4, p=0.94$ \\
\hline 10th & 1006 & 518 & 51.5 & 488 & 48.5 & \\
\hline 11th & 420 & 223 & 53.1 & 197 & 46.9 & \\
\hline 12th & 547 & 285 & 52.1 & 262 & 47.9 & \\
\hline School: & & & & & & Pearson $\chi^{2}$ \\
\hline GBSSS, Mahipalpur & 717 & 378 & 52.7 & 339 & 47.3 & $(5)=18.2, p=0.003$ \\
\hline GBSSS, Molarband & 551 & 315 & 57.2 & 236 & 42.8 & \\
\hline SBV, Molarband & 590 & 319 & 53.1 & 271 & 45.9 & \\
\hline GGSSS, Molarband & 726 & 338 & 46.6 & 388 & 53.4 & \\
\hline ASMS-SKV, Mahipalpur & 612 & 299 & 48.8 & 313 & 51.1 & \\
\hline SBV Co-Ed, Vasant Vihar & 391 & 208 & 53.2 & 183 & 46.8 & \\
\hline
\end{tabular}

referrals, $610(73.1 \%)$ consented to participate in the sensitisation trial and completed eligibility assessments for the host trial. The participation rate was significantly lower in the CC ( $\mathrm{n}=32$ of 55 referred in $\mathrm{CC}, 58.2 \%)$ as compared with the IC $(\mathrm{n}=578$ of 780 referred in IC, $74.1 \%$; Pearson $\left.\chi^{2}(1)=6.62, \mathrm{p}<0.001\right)$. Non-participation was associated with grade and school, but not with the age or gender of the student (table 2). Reported reasons for non-participation included no current problem/ problem resolved/ not otherwise interested $(n=8,34.8 \%$ in CC and $n=112,55.4 \%$ in IC), inability to read/comprehend the language of the intervention $(n=8,34.8 \%$ in CC and $n=19,9.4 \%$ in IC), referred by another party but not interested $(n=4,17.4 \%$ in $\mathrm{CC}$ and $\mathrm{n}=15,7.4 \%$ in IC) and 'lack of time' $(\mathrm{n}=1,0.5 \%$ in IC). Another $35(17.3 \%)$ adolescents, all from the IC, were not contacted as the

Table 2 Characteristics of the participants and non-participants for mental health threshold eligibility assessment

\begin{tabular}{|c|c|c|c|c|c|c|}
\hline & \multirow{2}{*}{$\begin{array}{l}\text { Total referred } \\
\mathrm{N}=835\end{array}$} & \multicolumn{2}{|c|}{ Trial participant } & \multicolumn{2}{|c|}{ Non-participant } & \multirow[b]{2}{*}{ Test statistic, $\mathbf{p}$ value } \\
\hline & & $\mathrm{N}=610$ & $\%$ & $\mathrm{~N}=\mathbf{2 2 5}$ & $\%$ & \\
\hline Age (mean, SD) & $\begin{array}{l}15.8 \text { years } \\
(0.06)\end{array}$ & \multicolumn{2}{|c|}{$\begin{array}{l}15.8 \text { years } \\
(0.06)\end{array}$} & \multicolumn{2}{|c|}{$\begin{array}{l}15.9 \text { years } \\
(0.13)\end{array}$} & $T$ test: $t(726)=0.2, p=0.84$ \\
\hline Gender & & & & & & Pearson $\chi^{2}(1)=0.02, p=0.90$ \\
\hline Female & 244 & 179 & 29.3 & 65 & 28.9 & \\
\hline 9th & 396 & 298 & 48.9 & 98 & 43.6 & \\
\hline 10th & 190 & 155 & 25.4 & 35 & 15.6 & \\
\hline 11th & 42 & 26 & 4.3 & 16 & 7.1 & \\
\hline 12th & 207 & 131 & 21.5 & 76 & 33.8 & \\
\hline SBV, Molarband & 202 & 122 & 20.0 & 80 & 35.6 & \\
\hline GGSSS, Molarband & 87 & 68 & 11.1 & 19 & 8.4 & \\
\hline ASMS-SKV, Mahipalpur & 80 & 38 & 6.2 & 42 & 18.7 & \\
\hline SBV Co-Ed, Vasant Vihar & 122 & 102 & 16.7 & 20 & 8.9 & \\
\hline
\end{tabular}


recruitment target of the host trial was achieved. Additionally, $n=3(13.0 \%)$ in the CC and $n=20,(9.9 \%)$ in the IC were not contactable.

\section{Intervention delivery}

The CC activities were conducted as per the protocol in most schools. The intervention supervisors met with the principals of five schools. The lay counsellors and researchers convened a total of 12 meetings with teachers ( 2 per school in 4 schools, 1 and 3 respectively in the remaining 2 schools; average duration $=15 \mathrm{~min}$ ). A total of 278 teachers $(66.0 \%$ of the total teacher body) participated. Six drop-boxes (one per school) were installed outside the counsellors' rooms, and 30 posters (five per school) were displayed in prominent locations.

The IC classroom sensitisation sessions were scheduled in two steps. The first was conducted in 35 classes at the beginning of the second time period with a mean duration of $23 \mathrm{~min}(\mathrm{SD}=0.81 \mathrm{~min}$; fidelity score $=1.88$, $\mathrm{SD}=0.04) ; 1136$ students $(61.2 \%$ of the relevant student population) attended these sessions. The remaining 35 classes received the classroom sensitisation sessions at the beginning of the third time period. The mean duration was $22.3 \mathrm{~min}$ ( $\mathrm{SD}=0.81 \mathrm{~min}$; fidelity score $=1.98, \mathrm{SD}=0.02$; t-test $(15)=-2.17, \mathrm{p}=0.05$, when compared with fidelity of the first step); 1211 students $(70.0 \%$ of relevant student population) attended the sessions. Regular school attendance was, however, not recorded for each of 3587 students during the trial.

\section{Effectiveness of the intervention}

The intervention was associated with a large effect on the primary outcome: out of 835 referrals, 55 (6.6\%) were generated from the CC, and $780(93.4 \%)$ from the IC. During the time periods when the classroom sensitisation sessions were delivered, the referral proportion rose with an OR of 111.36 (95\% CI 35.56 to 348.77, p<0.001) (figure 3). In the subsequent time period, the referrals dropped but continued to be significantly higher than in the CC (OR=5.80, 95\% CI 1.33 to 25.29; $\mathrm{p}=0.02)$. Sensitivity analysis was conducted for the primary outcome of referral proportion from the second period in line with the model used in the power calculation; the result $(\mathrm{OR}=134.67,95 \%$ CI 41.37 to $438.34, \mathrm{p}<0.001)$ was similar to the main analysis. We were not able to report the ICC for this study as the GEE analysis model for the primary outcome did not converge with an exchangeable correlation structure. School-based researchers and counsellors monitored for adverse events such as death, life-threatening events, clinical deterioration requiring hospitalisation or other specialist treatment, victimisation, sexual abuse, and chronic absenteeism as per the trial protocol. ${ }^{21}$ There were no adverse events recorded during the trial (table 3 ).

With regard to the secondary outcomes, out of the 610 referred adolescents for whom the data were available, $194(31.8 \%)$ fulfilled the criteria for mental health caseness. The proportion of cases of all students in the respective trial conditions was higher in the IC $(n=185$, $5.2 \%)$ as compared with the CC $(\mathrm{n}=9,0.3 \%)$, with $\mathrm{OR}=52.39,95 \%$ CI 12.49 to $219.66, \mathrm{p}<0.001$. However, there was no significant difference in the proportion of cases among the referred students in the two conditions: $28.1 \%$ ( $\mathrm{n}=9$ of 32 ) in the $\mathrm{CC}$ vs $32.0 \%$ ( $\mathrm{n}=185$ of 578) of the IC.

Overall, 814 adolescents (97.5\% of all referrals) were self-referred and 21 (2.5\% of all referrals) were referred by teachers. The proportion of self-referrals was significantly higher in the IC (IC=98.1\%, CC=89.1\%, Pearson $\left.\chi^{2}(1)=16.9213, p<0.001\right)$. We did not analyse this outcome any further, since the GEE model was unlikely to provide robust estimates considering the low number of teacherreferred adolescents.
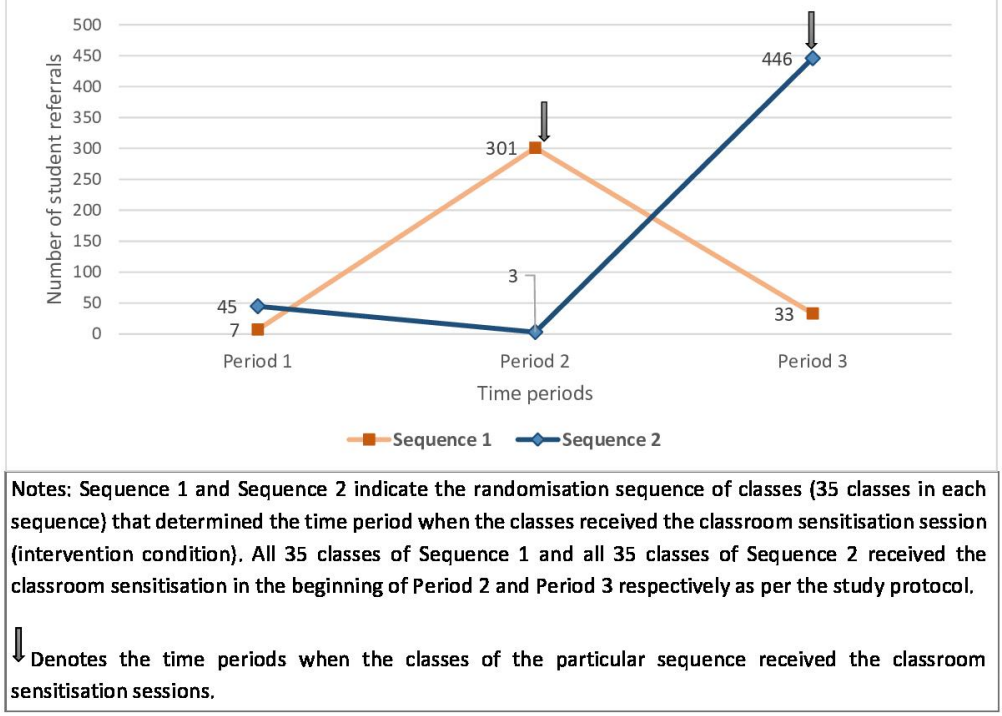

Figure 3 Student referrals received during the sensitisation trial. 


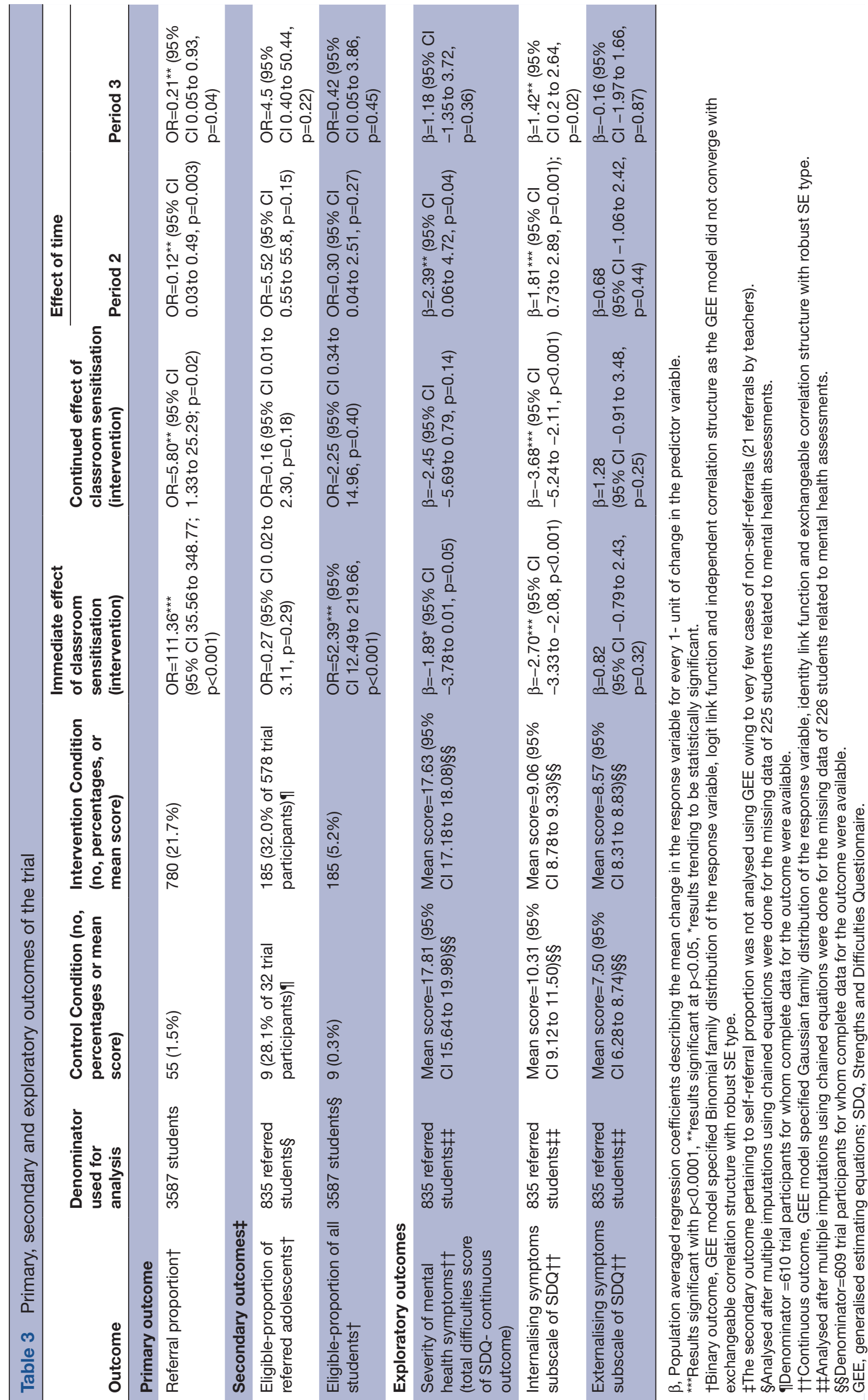


Considering exploratory outcomes, adolescents referred from the IC were characterised by lower SDQ Total Difficulties score by 1.89 points (95\% CI -3.78 to $0.01, \mathrm{p}=0.05$, adjusted for effects of time) and lower Internalising problem sub-scale score by 2.70 points; $95 \%$ CI -3.33 to $-2.08, \mathrm{p}<0.001$, adjusted for effects of time). There was no evidence that Externalising problem subscale scores were different between the two conditions.

\section{DISCUSSION}

This study evaluated the added impact of a classroom sensitisation session over and above whole-school sensitisation activities on demand for a school counselling service for adolescents in low-income secondary schools in New Delhi, India. The sensitisation trial was embedded in a host trial which evaluated the effectiveness of a problem-solving intervention delivered by lay counsellors. ${ }^{21} 22$ Thus, while the host trial sought to address supply side barriers (ie, lack of mental health care providers and contextually appropriate brief interventions), the sensitisation trial aimed to contribute to the scarce evidence on interventions that stimulate demand for mental healthcare in low-resourced contexts.

We observed that a single classroom sensitisation session covering common stressors and ways of coping, delivered by a lay counsellor, and consisting of a video presentation followed by a moderated group discussion, greatly increased referrals for mental healthcare compared with whole-school sensitisation activities alone. While the proportion of self-referrals was high in both conditions, it was significantly higher in the IC. The proportion of referrals who met mental health caseness criteria was similar in both conditions, but the fact that many more adolescents were referred from the IC meant that, overall, it accounted for a majority of the eligible referrals into the host trial.

Our findings are contrary to most previous studies which have found no effect of school-based mental health literacy interventions on adolescents' subsequent help-seeking, despite changes in knowledge and attitudes around mental healthcare in school settings. ${ }^{11-14}$ One exception was a recent US study by Lindow and colleagues, which demonstrated positive effects of the Youth Aware of Mental Health Intervention in schools on help-seeking from peers and school staff, but not mental health professionals for depression and suicidal thoughts, through an uncontrolled pretest/post-test design. ${ }^{30}$ Thus, our findings are unique in that they are based on a randomised controlled trial conducted in a low-resource context, where the intervention allowed for direct engagement of the service providers (counsellors) with adolescents through a structured classroom sensitisation session.

Our intervention was developed through multiple iterations and was specifically designed to address the barriers to help-seeking that we had identified in earlier formative research. ${ }^{19} 20$ The intervention not only aimed to facilitate a better understanding regarding mental health problems and the nature of available support in the participating schools, but also offered an opportunity for adolescents to discuss potential concerns such as confidentiality and familiarise themselves with counsellors in the process. It is notable that the most common reasons for non-participation differed between the two trial conditions, such that a greater proportion of non-participants in the IC reported an absence of mental health problems as the main reason for nonparticipation, as compared with those in the CC, who were more commonly either not interested after being referred by another party (such as teachers), or were unable to read or comprehend the language of the intervention (which was the same as the medium of instruction in all the selected schools). These suggest that the classroom sensitisation activities might have increased the awareness of symptoms of mental health problems and the availability of the counselling interventions, as well as improved acceptability of help-seeking.

A rapid referral/assessment system allowed adolescents to self-nominate immediately following the classroom sensitisation session, or shortly thereafter for students who preferred more discreet methods. Students who met the mental health caseness criteria were in turn provided with one of the formats for the low-intensity problem solving intervention (of the host trial) within the subsequent week. ${ }^{22}$ These features established an efficient link between the sensitisation session, referral process and on-site treatment provision. Thus, a key characteristic of our study design which may explain our findings is that the sensitisation programme was fully integrated with the counselling services in the school, in contrast to the other studies where a mental health intervention was often located in a separate setting. ${ }^{11-1430}$

The effects of the classroom intervention might have also been enhanced by other context-specific factors. As there was no existing counselling service available in the schools, the unmet need for psychological support among these adolescents was already high. It is notable that an overwhelming majority of the adolescents were self-referred across both the intervention and CC, as this indicates that the adolescents demonstrated personal agency in seeking psychological help. Potentially, cascading of information that was shared during the classroom session, with students who were absent, and also with students from the CC (cluster contamination) may have also influenced the self-referral process. Similarly the role of peer behaviour within the classroom sessions and subsequently may have also influenced this process, and warrants further investigation. ${ }^{5}$ Despite this, it cannot be ruled out that some students in need of psychological help may not have been referred at all, especially if they were unable to attend schools regularly.

The teacher-engagement component appeared to be low, despite the fact that two-thirds of teachers participated in the awareness meetings. This would help to explain the very small fraction of teacher-directed 
referrals. It is possible that a single meeting was not sufficient to identify and address potential barriers such as unfavourable attitudes of the teachers towards the school counselling programme or logistical reasons pertaining to lack of time and opportunities to know more about their students' mental health.

About two-thirds of referrals in both conditions did not meet the mental health caseness criteria, reflecting predefined clinical thresholds for symptom severity, functional impact and chronicity. In other words, while the addition of classroom sensitisation increased the total number of referrals and the total number of adolescents with mental health problems being referred, it was not more efficient than the whole-school sensitisation activities in increasing the proportion of referrals who, on the face of it, were most in need of mental healthcare. For a school-based programme, the implications of this finding are two-fold. First, the screening and assessment of large numbers of expected false positives may overwhelm the human resources allocated to the programme. Second, to ensure that school-based mental health services remain acceptable, the felt need of subthreshold referrals will need to be addressed by incorporating appropriate early interventions. For example, pilot work prior to the current trial suggested that adolescents expected school counsellors to provide practical guidance for mitigating their life problems (commonly related to academic performance, romantic relationships and conflicts with peers and family) ${ }^{19}$ In this context, offering low-cost 'bibliotherapy' or a brief single-session psychoeducation intervention containing practical tips for coping with common problems may be considered. ${ }^{22}$ Additionally, the use of digital technology to promote self-screening among adolescents and providing guidance may be considered. Adolescents referred for counselling from the IC reported symptoms that were less severe, particularly the internalising symptoms. which could be due to an increase in awareness of the availability and access to the counselling intervention in the school. This contrasts with the observation for externalising symptoms. However, this should be interpreted with caution considering these were exploratory outcomes and the trial may not be powered enough to detect these differences.

This trial is among the largest evaluations of a demandside intervention for adolescent mental healthcare anywhere in the world. It is also among the few trials to evaluate actual help-seeking behaviour. Among its other strengths are the inclusion of a sufficient number of clusters that provide adequate power to detect the effects of the intervention. The study used an active CC similar to many of the interventions tested in previous studies and employed minimal exclusion criteria for selecting the classes or referred adolescents for analysis. As the study was implemented in six government-run schools in low-income localities of New Delhi, India, the results are generalisable to similar settings in the real world. We also acknowledge several study limitations. Because of the short duration of the trial, we are unable to make inferences about the sustained effects of the intervention. That said, the declining secular trend for referrals following the intervention indicates the need for refresher sensitisation, perhaps once a semester/term. Although we did not assess economic costs, the brevity of the classroom sensitisation session and the fact that it was delivered by the same counsellor who subsequently treated eligible referrals (ie, requiring no additional human resources) would imply a strong likelihood of cost-effectiveness.

\section{CONCLUSION}

In conclusion, our study shows that demand for mental healthcare can be increased substantially in low-resource secondary schools through a single classroom sensitisation session, delivered by a lay counsellor, employing contextually relevant materials, in comparison to whole-school sensitisation activities alone. Such interventions should be twinned with school counselling services to enhance the effective coverage of mental healthcare for adolescents. Further research is needed to determine how school staff can be engaged more effectively to ensure wider and more efficient referral routes. Suitable interventions for adolescents who do not meet clinical thresholds also require further investigation, particularly in settings where mental health sensitisation generates demand that exceeds capacity for individual face-to-face interventions.

\section{Author affiliations}

${ }^{1}$ Department of Clinical, Neuro and Developmental Psychology, Faculty of Behavioural and Movement Sciences, Vrije Universiteit Amsterdam, Amsterdam, The Netherlands

${ }^{2}$ Sangath, Delhi, India

${ }^{3}$ PATH, Delhi, India

${ }^{4} \mathrm{GGZ}$ inGeest, Amsterdam, The Netherlands

${ }^{5}$ Amsterdam UMC, Vrije Universiteit, Psychiatry, Amsterdam Public Health Research Institute, Amsterdam, The Netherlands

${ }^{6}$ School of Psychology, University of Sussex, Brighton, UK

${ }^{7}$ Sangath, Porvorim, Goa, India

${ }^{8}$ Department of Clinical, Neuro and Developmental Psychology, Amsterdam Public Health Research Institute, Amsterdam, The Netherlands

${ }^{9}$ Department of Global Health and Social Medicine, Harvard Medical School, Boston, Massachusetts, USA

${ }^{10}$ Department of Global Health and Population, Harvard T.H. Chan School of Public Health, Boston, Massachusetts, USA

Acknowledgements We gratefully acknowledge the contribution of Jennifer Thompson and Helen Weiss in reviewing the statistical analysis plan.

Contributors RP drafted the report, which all authors reviewed and approved. $\mathrm{AH}$, $\mathrm{DM}, \mathrm{PC}$ and VP critically revised the content of the report. RP, AH, JR, PC, DM and VP designed the trial. DM, RSh, KM, BB and VP devised the intervention content and data collection instruments. RP, DM, RSa and RSh were responsible for trial conduct. $\mathrm{BB}$ and $\mathrm{RP}$ were responsible for database design and management. RP and $\mathrm{AH}$ did the statistical analyses.

Funding This study was supported by a Principal Research Fellowship awarded to Vikram Patel by the Wellcome Trust (Grant no. 106919/A/15/Z).

Disclaimer The funding agency has no role in the design of the study and in data collection, or the writing of the manuscript.

Competing interests None declared.

Patient and public involvement Patients and/or the public were involved in the design, or conduct, or reporting, or dissemination plans of this research. Refer to the Methods section for further details. 
Patient consent for publication Not required.

Ethics approval Ethics approval was obtained from the Institutional Review Boards of Sangath and Harvard Medical School.

Provenance and peer review Not commissioned; externally peer reviewed.

Data availability statement Data are available on request. Anonymised participant data, data dictionary and CRFs will be made available by 12 months after trial completion at: https://datacompass.Ishtm.ac.uk/. Data will be shared after approval by the $\mathrm{Pl}$, following a reasonable request submitted through the above site. The study protocol and statistical analysis plan are publicly available at: https://clinicaltrials.gov/ct2/show/NCT03633916https://clinicaltrials.gov/ ProvidedDocs/16/NCT03633916/SAP_000.pdf.

Open access This is an open access article distributed in accordance with the Creative Commons Attribution Non Commercial (CC BY-NC 4.0) license, which permits others to distribute, remix, adapt, build upon this work non-commercially, and license their derivative works on different terms, provided the original work is properly cited, appropriate credit is given, any changes made indicated, and the use is non-commercial. See: http://creativecommons.org/licenses/by-nc/4.0/.

Author note This paper is dedicated to the memory of Dr Jeroen Ruwaard.

ORCID iD

Rachana Parikh http://orcid.org/0000-0002-0761-5513

\section{REFERENCES}

1 Adhikari RP, Upadhaya N, Suwal BR. Factors associated with perceived psychosocial problems and help-seeking practices among adolescents in Nepal. J Population Soc Stud 2017;25:1-10.

2 Dardas LA, Silva SG, van de Water B, et al. Psychosocial correlates of Jordanian adolescents' help-seeking intentions for depression: findings from a nationally representative school survey. J Sch Nurs 2019;35:117-27.

3 Ibrahim N, Amit N, Shahar S, et al. Do depression literacy, mental illness beliefs and stigma influence mental health help-seeking attitude? A cross-sectional study of secondary school and university students from B40 households in Malaysia. BMC Public Health 2019;19:544-44

4 Ogorchukwu JM, Sekaran VC, Nair S, et al. Mental health literacy among late adolescents in South India: what they know and what attitudes drive them. Indian J Psychol Med 2016;38:234-41.

5 Radez J, Reardon T, Creswell C, et al. Why do children and adolescents (not) seek and access professional help for their mental health problems? A systematic review of quantitative and qualitative studies. Eur Child Adolesc Psychiatry 2021;30:183-211.

6 Gronholm PC, Nye E, Michelson D. Stigma related to targeted school-based mental health interventions: a systematic review of qualitative evidence. J Affect Disord 2018;240:17-26.

7 Xu Z, Huang F, Kösters M, et al. Effectiveness of interventions to promote help-seeking for mental health problems: systematic review and meta-analysis. Psychol Med 2018;48:2658-67.

8 Salerno JP. Effectiveness of universal school-based mental health awareness programs among youth in the United States: a systematic review. J Sch Health 2016;86:922-31.

9 Wei Y, Hayden JA, Kutcher S, et al. The effectiveness of school mental health literacy programs to address knowledge, attitudes and help seeking among youth. Early Interv Psychiatry 2013;7:109-21.

10 Singh S, Zaki RA, Farid NDN. A systematic review of depression literacy: knowledge, help-seeking and stigmatising attitudes among adolescents. J Adolesc 2019;74:154-72.

11 Sharpe H, Patalay P, Vostanis P, et al. Use, acceptability and impact of booklets designed to support mental health self-management and help seeking in schools: results of a large randomised controlled trial in England. Eur Child Adolesc Psychiatry 2017;26:315-24.

12 Vieland V, Whittle B, Garland A, et al. The impact of curriculumbased suicide prevention programs for teenagers: an 18-month follow-up. J Am Acad Child Adolesc Psychiatry 1991;30:811-5.

13 Spirito A, Overholser J, Ashworth S, et al. Evaluation of a suicide awareness curriculum for high school students. J Am Acad Child Adolesc Psychiatry 1988;27:705-11.

14 Aseltine $\mathrm{RH}$, James A, Schilling EA, et al. Evaluating the SOSsuicide prevention program: a replication and extension. BMC Public Health 2007;7:161.

15 Roy K, Shinde S, Sarkar BK, et al. India's response to adolescent mental health: a policy review and stakeholder analysis. Soc Psychiatry Psychiatr Epidemiol 2019;54:405-14.

16 Gururaj G, Varghese M, Benegal V. National mental health survey of India, 2015-16: summary. Bengaluru: National Institute of Mental Health and Neuro Sciences, 2016.

17 Michelson D, Malik K, Krishna M, et al. Development of a transdiagnostic, low-intensity, psychological intervention for common adolescent mental health problems in Indian secondary schools. Behav Res Ther 2020;130:103439.

18 Chorpita BF, Daleiden EL, Malik K, et al. Design process and protocol description for a multi-problem mental health intervention within a stepped care approach for adolescents in India. Behav Res Ther 2020;133:103698.

19 Parikh R, Michelson D, Sapru M, et al. Priorities and preferences for school-based mental health services in India: a multi-stakeholder study with adolescents, parents, school staff, and mental health providers. Glob Ment Health 2019;6:e18.

20 Parikh R, Sapru M, Krishna M, et al. "It is like a mind attack": stress and coping among urban school-going adolescents in India. BMC Psychol 2019;7:31.

21 Parikh R, Michelson D, Malik K, et al. The effectiveness of a lowintensity problem-solving intervention for common adolescent mental health problems in New Delhi, India: protocol for a schoolbased, individually randomized controlled trial with an embedded stepped-wedge, cluster randomized controlled recruitment trial. Trials 2019;20:568.

22 Michelson D, Malik K, Parikh R, et al. Effectiveness of a brief lay counsellor-delivered, problem-solving intervention for adolescent mental health problems in urban, low-income schools in India: a randomised controlled trial. Lancet Child Adolesc Health 2020;4:571-82.

23 Hussey MA, Hughes JP. Design and analysis of stepped wedge cluster randomized trials. Contemp Clin Trials 2007;28:182-91.

24 Goodman R, Ford T, Simmons $\mathrm{H}$, et al. Using the strengths and difficulties questionnaire (SDQ) to screen for child psychiatric disorders in a community sample. Br J Psychiatry 2000;177:534-9.

25 Bhola P, Sathyanarayanan V, Rekha DP, et al. Assessment of selfreported emotional and behavioral difficulties among pre-university college students in Bangalore, India. Indian J Community Med 2016;41:146-50.

26 Srinath S, Kandasamy P, Golhar TS. Epidemiology of child and adolescent mental health disorders in Asia. Curr Opin Psychiatry 2010;23:330-6.

27 Thompson JA, Davey C, Fielding K, et al. Robust analysis of stepped wedge trials using cluster-level summaries within periods. Stat Med 2018;37:2487-500

28 White IR, Royston P, Wood AM. Multiple imputation using chained equations: issues and guidance for practice. Stat Med 2011;30:377-99.

29 Barker D, McElduff P, D'Este C, et al. Stepped wedge cluster randomised trials: a review of the statistical methodology used and available. BMC Med Res Methodol 2016;16:69.

30 Lindow JC, Hughes JL, South C, et al. The youth aware of mental health intervention: impact on help seeking, mental health knowledge, and stigma in U.S. adolescents. J Adolesc Health 2020;67:101-7 